\title{
Fortune and reversals of fortune in visual search: Reward contingencies for pop-out targets affect search efficiency and target repetition effects
}

\author{
ÁRNI KRISTJÁNSSON \\ University of Iceland, Reykjavik, Iceland \\ and University College London, London, England \\ Ólafía SiguRJónSDótTIR \\ University of Iceland, Reykjavik, Iceland \\ AND \\ JON DRIVER \\ University College London, London, England
}

\begin{abstract}
Rewards have long been known to modulate overt behavior. But their possible impact on attentional and perceptual processes is less well documented. Here, we study whether the (changeable) reward level associated with two different pop-out targets might affect visual search and trial-to-trial target repetition effects (see Maljkovic \& Nakayama, 1994). Observers searched for a target diamond shape with a singleton color among distractor diamond shapes of another color (e.g., green among red or vice versa) and then judged whether the target had a notch at its top or bottom. Correct judgments led to reward, with symbolic feedback indicating this immediately; actual rewards accumulated for receipt at study end. One particular target color led to a higher (10:1) reward for $75 \%$ of its correct judgments, whereas the other singleton target color (counterbalanced over participants) yielded the higher reward on only $25 \%$ of the trials. We measured search performance in terms of inverse efficiency (response time/proportion correct). The reward schedules not only led to better performance overall for the more rewarding target color, but also increased trial-to-trial priming for successively repeated targets in that color. The actual level of reward received on the preceding trial affected this, as did (orthogonally) the likely level. When reward schedules were reversed within blocks, without explicit instruction, corresponding reversal of the impact on search performance emerged within around 6 trials, asymptoting at around 15 trials, apparently without the observers' explicit knowledge of the contingency. These results establish that pop-out search and target repetition effects can be influenced by target reward levels, with search performance and repetition effects dynamically tracking changes in reward contingency.
\end{abstract}

Visual search is a widely studied paradigm involving laboratory versions of the common real-world problem of finding an item of current interest among a cluttered visual scene containing many items (for reviews, see Kristjánsson, 2006; Kristjánsson \& Campana, 2010; Müller \& Krummenacher, 2006; Wolfe, 1998; Wolfe \& Horowitz, 2004). Early accounts of visual search (e.g., Julesz, 1984; Neisser, 1967; Treisman \& Gelade, 1980) mainly emphasized the role of bottom-up feature contrasts in determining how well a target stands out from surrounding nontargets.

Subsequent research has shown that the effects of such bottom-up saliency can be modulated by other factors, such as the behavioral importance of the stimuli (Ivry \&
Cohen, 1990; Müller, Heller, \& Ziegler, 1995; D. Wang, Kristjánsson, \& Nakayama, 2005; Q. Wang, Cavanagh, \& Green, 1994; Wolfe, Cave, \& Franzel, 1989, although such top-down modulations seemingly have their limits: see Van der Stigchel et al., 2009), or by what has occurred on previous trials (e.g., Becker, 2008; Geyer, Müller, \& Krummenacher, 2006; Kristjánsson \& Driver, 2008; Lamy, Antebi, Aviani, \& Carmel, 2008; Maljkovic \& Nakayama, 2000; Olivers \& Meeter, 2006; Sigurdardottir, Kristjánsson, \& Driver, 2008; see Kristjánsson, 2008, for a review). Observers typically perform better in search when the same target is repeated on successive trials, as compared with when the target changes, even for so-called pop-out

Á. Kristjánsson, ak@hi.is 
targets that differ from nontargets in a salient singleton feature (e.g., red among green), leading to the phenomenon of priming of pop-out when singleton properties are repeated (Maljkovic \& Nakayama, 1994).

Kiss, Driver, and Eimer (2009) recently reported that search was faster for pop-out targets associated with higher reward (as symbolically indicated by points that participants considered as relating to eventual financial reward). Della Libera and Chelazzi (2006) reported potentially related effects of reward on distractor rejection. The specific phenomenon of priming of pop-out (Maljkovic \& Nakayama, 1994) has been considered largely impervious to top-down goal settings (e.g., Kristjánsson, 2008; Kristjánsson \& Nakayama, 2003; Maljkovic \& Nakayama, 1994) and is now known to affect neural activity in the early visual cortex (e.g., Geng et al., 2006; Kristjánsson, Vuilleumier, Schwartz, Macaluso, \& Driver, 2007). Here, we studied systematically whether priming of pop-out can nevertheless be modulated by reward levels associated with different pop-out targets (for potentially related but independent conference abstracts, see Hickey \& Theeuwes, 2008; Kristjánsson, Sigurjónsdóttir, \& Driver, 2009; Shen \& Chun, 2009). Importantly, our design allowed us to distinguish the likely reward level from that actually obtained on some trials. In a second experiment, we examined the impact of reversing reward contingencies unpredictably (see Sugrue, Corrado, \& Newsome, 2004) for our singleton targets.

\section{EXPERIMENT 1}

\section{Method}

Participants. Eight participants (21-45 years of age; six of them female) underwent four blocks of 100 trials. All reported normal or corrected-to-normal visual acuity. Their color vision was normal, as tested with Ishihara color plates.

Equipment. The search display was programmed in $\mathrm{C}$ and presented on a $75-\mathrm{Hz}$ CRT controlled by a $400-\mathrm{MHz}$ G4 Apple computer.

Stimuli and Procedure. Each trial started with the presentation of a white (56.6- $\left.\mathrm{cdm}^{-2}\right)$ fixation cross for 1,200-1,700 msec on a dark background $\left(0.8 \mathrm{cdm}^{-2}\right)$, followed by a search display containing three diamond shapes (each sized $2.4^{\circ} \times 2.4^{\circ}$, at $4.05^{\circ}$ from screen center; see the left panels of Figures 1A and 1B) on the same background. Observers searched for the oddly colored diamond, either a red $\left(14.8 \mathrm{cdm}^{-2}\right)$ target among two green distractors $\left(13.9 \mathrm{cdm}^{-2}\right)$ or vice versa. Observers judged (by keypress) whether the singleton diamond had a notch at the top or the bottom (see also Kiss et al., 2009; Kristjánsson, Vuilleumier, Malhotra, Husain, \& Driver, 2005; Kristjánsson et al., 2007). Whether the singleton target was red or green on any given trial was determined completely randomly. This task is, in essence, similar to that introduced by Bravo and Nakayama (1992), as subsequently used by Maljkovic and Nakayama (1994) to demonstrate priming of pop-out, and is equivalent to that used by Kristjánsson et al. (2005; Kristjánsson et al., 2007).

Following each trial, the number of points earned on that trial was displayed in white Helvetica, digit height 48' (see the right panels of Figures 1A and 1B). For half the participants, a correct response for a red target trial yielded 10 points on $75 \%$ of such trials, 1 point for the other $25 \%$, but vice versa for the green targets. For the other participants, this was reversed. Incorrect responses gave 0 points. To emphasize the reward outcomes, auditory feedback was provided.
If the reward was 10 points, a sequence of four sounds was heard (each for $15 \mathrm{msec}$, no interval) that successively rose in frequency (range, $261-523 \mathrm{~Hz}$ in steps of $86 \mathrm{~Hz}$; i.e., middle $\mathrm{C}$ to $\mathrm{C}_{5}$ ) from one to the next (yielding a sound reminiscent of the "bank" sound heard on many slot machines). A high-frequency sinusoidal tone $(523 \mathrm{~Hz})$ was heard for $60 \mathrm{msec}$ if the reward was 1 point, or a lower sinusoidal tone $(65 \mathrm{~Hz})$ was heard if the response was incorrect.

To encourage fast as well as accurate responding, the participants were told before data collection that if they were the fastest of the 8 observers in the study, they could double their eventual earnings, but they still had strong motivation to be accurate, since no reward was given for incorrect responses. The reward points represented Icelandic krona (one for one), and at study end, the participants received a gift certificate for the university bookstore in the amount earned. The obtained totals (prior to speed considerations) ranged from 1,903 to 2,225 ISK, and the fastest participant doubled his or her earned amount, as explained above. The observers were told that they would be rewarded for fast and accurate performance, but without the relative values of specific target colors being explained. Although the subjective value of gift certificates might potentially vary between participants, we note that the same could apply for purely financial reward.

\section{Results and Discussion}

Figure 1C shows inverse efficiency plots (response times [RTs] divided by proportion correct, a standard way to combine RT with accuracy; see, e.g., Townsend \& Ashby, 1983) as a function of successive trial-to-trial repetitions of the target color, separately for the highreward and low-reward cases (pooled across actual color, as counterbalanced over participants). The corresponding RT data are shown below in Figure 1D. Error rates appear in Table 1.

A 2 (reward level) $\times 6$ (zero through to five or more successive repetitions of target color) repeated measures ANOVA on the inverse-efficiency scores revealed a clear main effect of reward level $[F(1,7)=17.6, p=.004]$, with better performance overall for high-reward targets, replicating the analogous finding of Kiss et al. (2009). Note that our results (Figures 1C and 1D) suggest that this main effect can be qualified by an interaction with repetition (see below). Second, there was a main effect of repeating target color $[F(5,35)=12.7, p<.001]$, with better performance for more repeats, replicating the priming-of-pop-out findings of Maljkovic and Nakayama (1994) and the streak phenomenon identified by Kristjánsson and colleagues (e.g., Kristjánsson, 2009; Kristjánsson, Mackeben, \& Nakayama, 2001). The critical result for our purposes was a significant interaction between reward level and target color repetition $[F(5,35)=7.8, p<.001]$. The benefit of successive target repetitions was larger for the more highly rewarded target color (see Figures 1C and 1D). There were no significant terms in a two-way ANOVA on the error rates (all $F_{\mathrm{s}}<1.5$, n.s.).

We also analyzed the target repetition effects as a function of whether the reward level actually received on the preceding trial was the usual (likely) reward level for a given color or not (see Figure 2). Note that the usual reward for a given color was obtained on only $75 \%$ of correct trials, with the other reward level being obtained instead on the remaining $25 \%$. A 2 (actual reward level received) $\times$ 
A

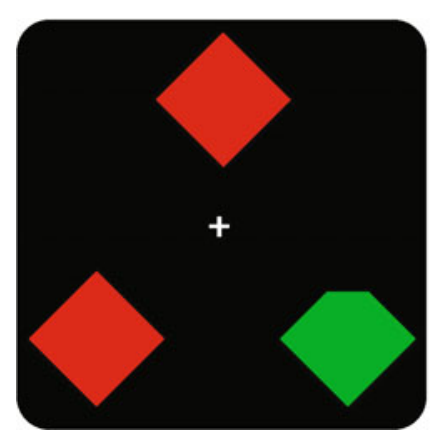

High Reward

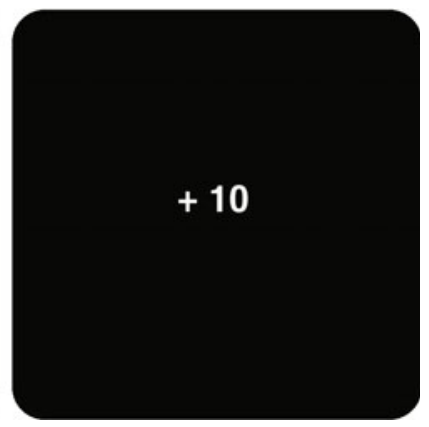

B

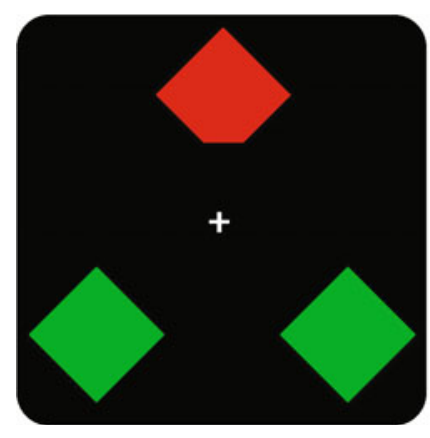

Low Reward

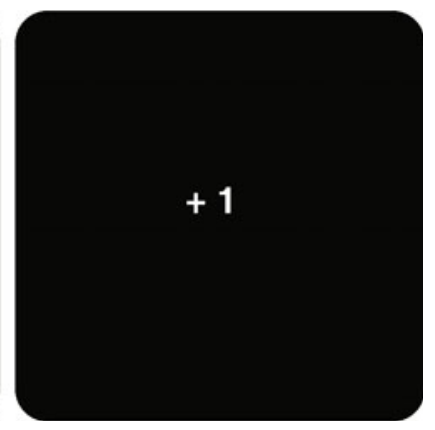

\section{Inverse Efficiency (Experiment 1)}

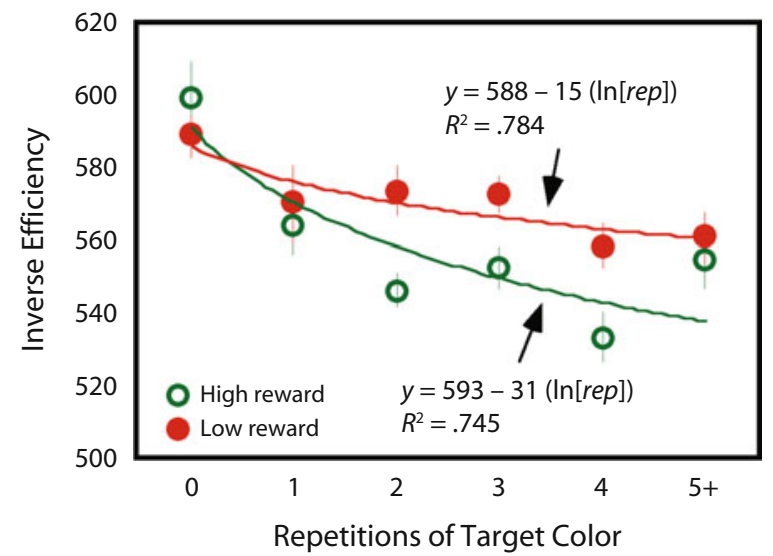

D Response Times (Experiment 1)

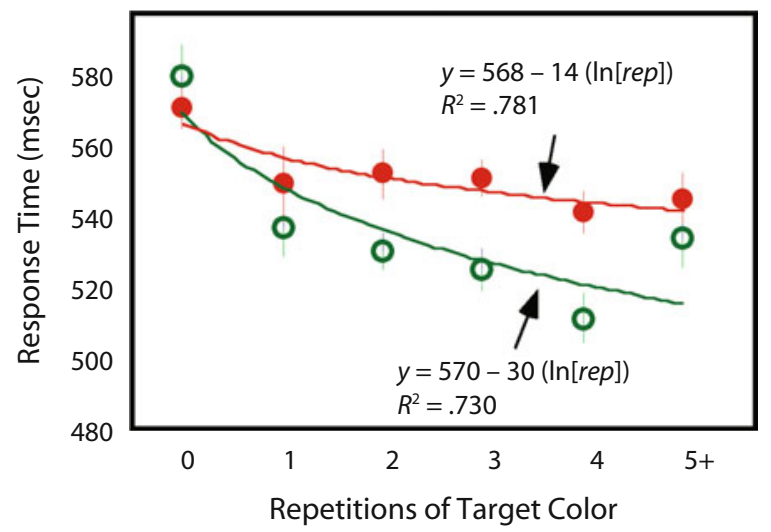

Figure 1. Example displays and results for Experiment 1. (A) Example of a high-reward trial (in this case, for a green [light gray in printed edition of this article] target, with the display in the left panel and the feedback received for correct judgment on the right). (B) Example of a low-reward trial (red [dark gray in print] target in this particular example). (C) Plot of inverse efficiency (response time [RT]/proportion correct) for target color repetition across successive trials, shown separately for high- and low-reward targets (actual color counterbalanced across participants). (D) Corresponding RT results. The fitted lines show decreasing logarithmic functions fit to the data to highlight the pattern of results ("rep" denotes number of repetitions of a target color, as shown along the $x$-axis). These logarithmic fits provide a useful summary of the data, as indicated by the $R^{2}$ values given. Note the enhanced performance for the high-reward target color, especially when repeated. Error bars represent the $S E M$ s.

2 (likely reward level for a given color in each participant) ANOVA on the inverse efficiency target repetition effects (nonrepeat minus repeat trials) showed a main effect of actual reward level received on trial $n$ for the target color repetition effect on trial $n+1[F(1,7)=7.3, p<.05]$, in

Table 1

Percentages of Errors in the Two Experiments As a Function of Reward Schedule and Successive Repetitions of Target Color

\begin{tabular}{cccccc}
\hline & \multicolumn{2}{c}{ Experiment 1 } & & \multicolumn{2}{c}{ Experiment 2 } \\
\cline { 2 - 3 } \cline { 5 - 6 } $\begin{array}{c}\text { Repetitions of } \\
\text { Target Color }\end{array}$ & $\begin{array}{c}\text { High } \\
\text { Reward }\end{array}$ & $\begin{array}{c}\text { Low } \\
\text { Reward }\end{array}$ & & $\begin{array}{c}\text { High } \\
\text { Reward }\end{array}$ & $\begin{array}{c}\text { Low } \\
\text { Reward }\end{array}$ \\
\hline 0 & 3.6 & 3.1 & & 2.9 & 3.2 \\
1 & 4.9 & 3.7 & & 3.3 & 2.5 \\
2 & 3.0 & 3.9 & & 3.8 & 2.7 \\
3 & 5.0 & 3.9 & & 2.1 & 3.8 \\
4 & 4.1 & 3.2 & & 4.5 & 3.1 \\
$5+$ & 3.8 & 2.9 & & 2.2 & 2.0 \\
\hline
\end{tabular}

addition to a main effect of the likely $(75 \%)$ reward level for that color $[F(1,7)=5.9, p<.05]$, with no interaction $[F(1,7)=1.9$, n.s.]. Overall, the results in Experiment 1 clearly show that reward level modulates priming of pop-out on a trial-by-trial basis. Both the actual reward received and the likely level of that reward for a given target color have an impact.

To sum up, Experiment 1 provided an initial demonstration that target repetition effects corresponding to priming of pop-out, long considered to be neither under voluntary control nor modulated by task priorities (e.g., Kristjánsson \& Nakayama, 2003; Maljkovic \& Nakayama, 1994; but see Fecteau, 2007), can be substantially modulated by the reward level associated with the different singleton colors, in terms of both the likely reward level and the level of reward actually received on a given trial. Our second experiment investigated the impact of unexpectedly reversing the reward contingencies within a trial block. 


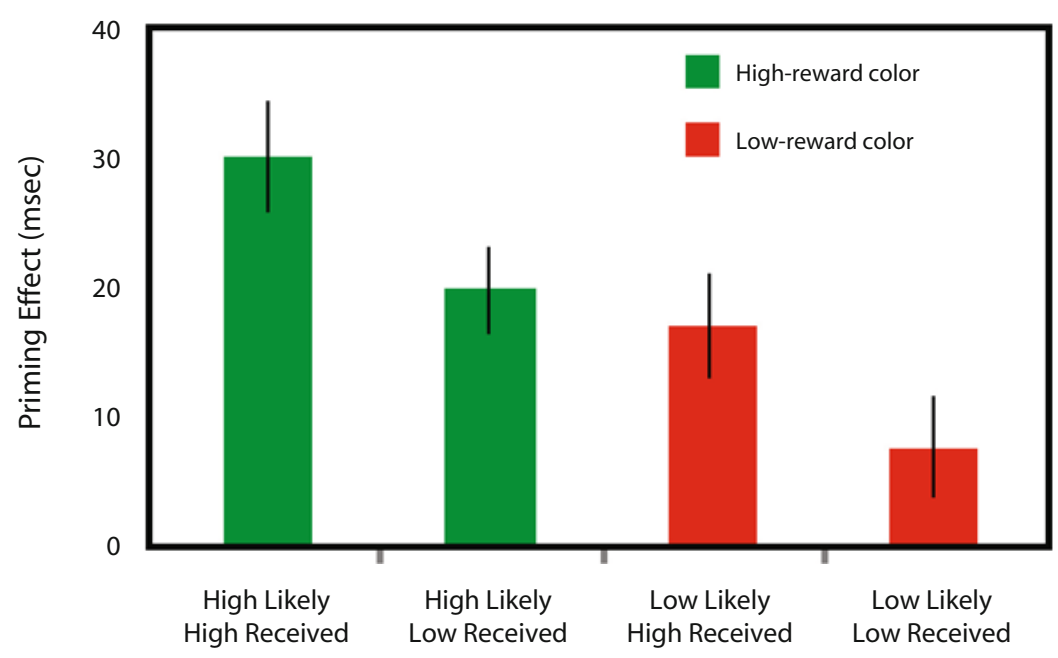

Figure 2. Target repetition effects from the preceding to the current trial (plotted here in terms of inverse-efficiency differences for nonrepeat minus repeat trials, along the $y$-axis) depend on both the likely (i.e., $75 \%$ ) level of reward and the actual reward received for the preceding trial. In the bar plot, target repetition effects are shown separately for the target color that was likely (75\%) to yield a high reward (indicated with green [light gray in printed edition] bars here, but actual target color was counterbalanced across subjects) versus for the target color that was more likely (75\%) to yield a low reward instead (indicated with red [dark gray in print] bars here). For either type of target, target repetition effects are shown separately as a function of the level of reward actually received on the preceding trial. The error bars represent one $S E M$ in either direction.

\section{EXPERIMENT 2}

The procedure was similar to that in Experiment 1, except that the reward contingencies could now reverse unpredictably during blocks, without the observers being instructed on this.

\section{Method}

Participants. Fifteen new observers ( 9 of them female; ages, 21-36 years) participated. Each took part in three blocks of 200 trials, with a short break in between. All had normal or corrected-tonormal visual acuity and intact color vision.

Procedure. During each 200-trial block, one randomly chosen target color (red or green) was designated as high reward at block start (yielding a high reward for $75 \%$ of correct judgments, as before), the other color as low reward. But during the course of the 200 trials, this reward schedule could now reverse unpredictably, three times per block (at randomly determined points between Trials $21-60,81-120$, or 141-180). As before, the observers were simply told that they would be rewarded for fast and accurate performance, without comment on the relative (and now reversible) values of the two singleton colors.

\section{Results and Discussion}

Figure 3A shows an inverse-efficiency plot (RT/ proportion correct) as a function of successive repetitions of the same target color, separately for the (currently) high-reward versus low-reward colors. The RT data are plotted in Figure 3B, and error rates are given in Table 1.

A $2 \times 6$ repeated measures ANOVA on the inverseefficiency scores again showed a clear main effect of reward level $[F(1,14)=23.8, p<.001]$ and of target color repetition across successive trials $[F(5,70)=14.7, p<$
.001]. More important, reward level and repetition interacted $[F(5,70)=9.3, p<.001]$, showing a bigger effect of target repetition for the (currently) high-reward color once again. A comparable ANOVA on error rates yielded no significant terms (all $F_{\mathrm{S}}<1$, n.s.).

Figure 4A shows mean inverse efficiency across the 15 observers, for trials preceding, during, and after an unpredictable reversal of the reward schedule, for the different (counterbalanced) target colors. Overall performance clearly "tracks" the reversal in reward contingency. The confidence intervals on the performance measure no longer overlap (indicating significant differences at $\alpha=.05$ ) with the prereversal levels, from the sixth trial after a reversal onward. Figure 4B shows the results of an analogous analysis, but now for the average priming-of-pop-out effect (repeats of target color compared with nonrepeats, in inverse efficiency). Positive values on the $y$-axis now indicate an advantage for repetition of target color. Analogously to overall performance (Figure 4A), target repetition effects also clearly track the reversal in reward contingency, with postreversal scores no longer overlapping (again indicating a significant difference) with prereversal, from the sixth trial after a reversal onward once again.

As in Experiment 1, we also assessed the impact of the reward actually received on a given trial, as distinct from the reward that was likely for a given target color, for priming of pop-out. We now did this separately for the early part of each block prior to a reversal in reward contingency versus the later part of a block subsequent to a reversal (since which color was likely [75\%] to yield high or low rewards reversed between these two parts of each block). 


\section{A Inverse Efficiency (Experiment 2)}

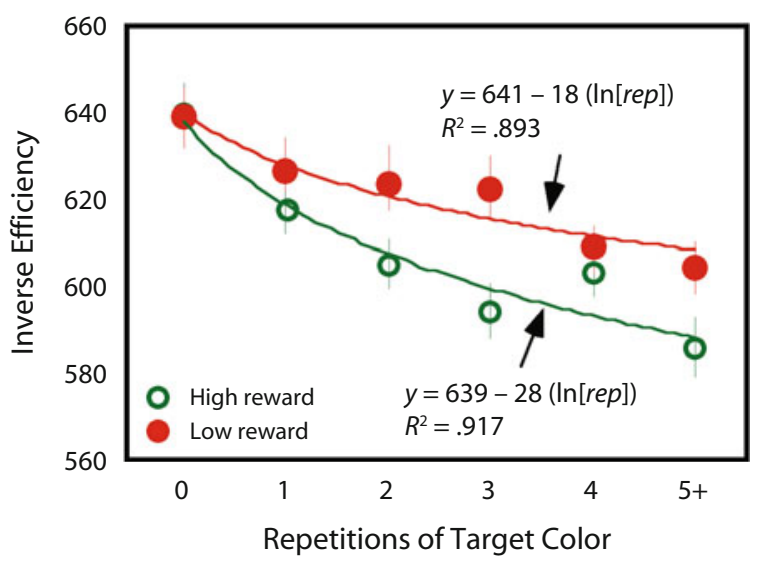

B Response Times (Experiment 2)

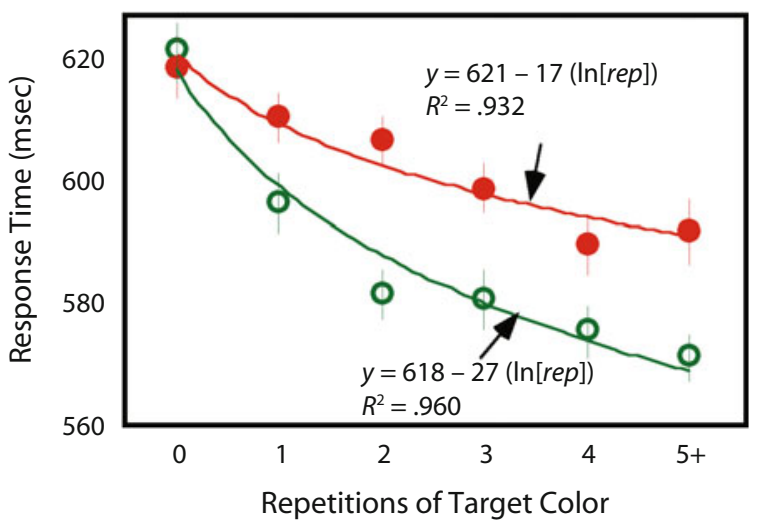

Figure 3. Results from Experiment 2. (A) Inverse efficiency (response time $[\mathrm{RT}] /$ proportion correct) as a function of successive repetitions of target color, separately for currently high- or low-reward target colors (now counterbalanced both across and within participants). (B) RT data from the same experiment. The fitted lines show decreasing logarithmic fits to the results to highlight the pattern of results ("rep" in the equations denotes repetitions of target color, the values on the abscissa). Note once again the better performance for the (currently) high-reward target color, especially when repeated. Error bars represent $S E M$ s.

A three-way $(2 \times 2 \times 2)$ ANOVA on inverse-efficiency scores had the factors of actual reward and usual (i.e., currently $75 \%$ likely) reward, plus an additional pre- versus postreversal factor. There were significant main effects of all three factors (all $F \mathrm{~s}>5.3$, all $p \mathrm{~s}<.05$ ), as well as a significant interaction $[F(1,14)=4.9, p<.05]$ between usual reward and pre- versus postreversal. This interaction reflected a larger impact of the usual (i.e., currently likely) reward prior to $[t(14)=7.9, p<.01]$, rather than after $[t(14)=1.5$, n.s. $]$, the reversal in reward contingency. This presumably arose because the usual (75\% likely) level of reward was less clear in the trials immediately following the reversal. The data in Figure 4 indicate that following the reversal in contingency, performance stabilized under the new regime within approximately 15 trials. We therefore implemented a similar three-way ANOVA, but now after removing data for the first 15 trials following a reversal. The only significant terms in the resulting three-way ANOVA were now just the main effects of actual reward received $[F(1,14)=12.8, p<.01]$ and the usual (i.e., currently $75 \%$ likely) reward $[F(1,14)=9.6, p<.01]$. This pattern of noninteracting effects of actual reward and likely reward resembles the result in Experiment 1 (cf. Figure 2), where the usual reward levels remained stable throughout. The mean inverse-efficiency primingof-pop-out scores (after excluding the 15 trials immediately following the reversal in contingency) were $28 \mathrm{msec}$ for high-actual/high-likely, 16 for low-actual/high-likely, 20 for low-likely/high-actual, and 10 for low-likely/lowactual, revealing a pattern similar to that in Experiment 1 (cf. Figure 2). Although a reviewer asked what the results might look like if we analyzed further back than for just trial $n-1$, we did not have sufficient power to implement this here (cf., e.g., Lau \& Glimcher, 2005).

We asked our participants in debriefing whether they had noticed anything systematic concerning the reward schedules. Thirteen of the 15 participants told us that they had not noticed any pattern and thought the reward schedules were random. Although the remaining 2 felt that there might be a pattern, they could not describe it.

\section{GENERAL DISCUSSION}

Our experiments indicate not only that visual search targets associated with higher reward levels can lead to better overall performance (see also Kiss et al., 2009, who reported that this can relate to an enhanced N2pc ERP component), but also show that priming of pop-out, for successive repetitions of singleton target color (Kristjánsson \& Driver, 2008; Kristjánsson, Ingvarsdóttir, \& Teitsdóttir, 2008; Maljkovic \& Nakayama, 1994, 2000; Sigurdardottir et al., 2008), can be significantly enhanced for targets associated with higher reward. Priming of pop-out had previously been considered largely immune to voluntary control or task priorities and to involve a low-level passive memory mechanism (Goolsby \& Suzuki, 2001; Kristjánsson \& Nakayama, 2003; Maljkovic \& Nakayama, 2000) likely to reflect activity modulations in the early visual cortex (Geng et al., 2006; Kristjánsson et al., 2005; Kristjánsson et al., 2007; see also Campana, Cowey, \& Walsh, 2002, 2006). Here, we found that target repetition effects in search are substantially influenced by the reward level associated with particular targets (and by the level actually received; see Figure 2). Moreover, the extent of priming of pop-out dynamically tracked unpredictable reversals in these reward levels (see Figure 4B), leading to significant changes within 6 trials of such a reversal and apparently stabilizing within approximately 15 trials.

Rather than reflecting a purely passive visual memory, target repetition effects in search can evidently adapt to changing reward contingencies, to enhance repeated searches for the currently most rewarding visual targets. The potential adaptive significance of this for any animal seeking to maximize its reward seems self-evident, although it would be interesting for future research to test the extent to which the present reward effects lead to optimal 
A Inverse Efficiency With Changes in Reward Schedule (Experiment 2)

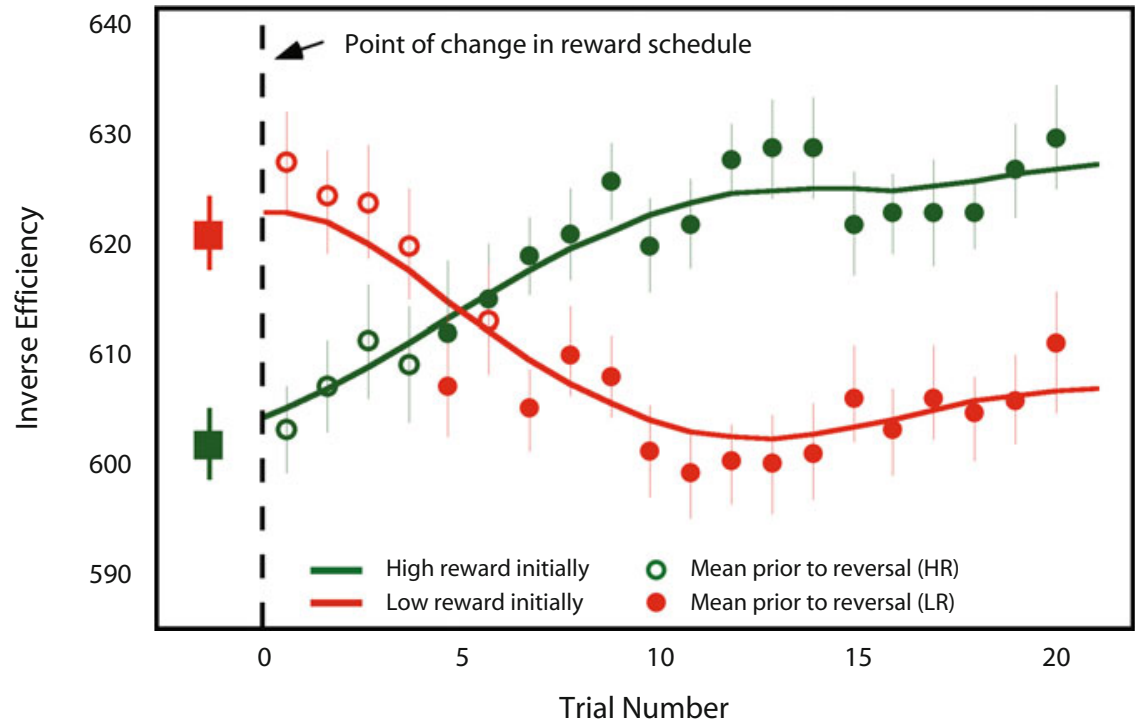

B Mean Priming Effects With Changes in Reward Schedule (Experiment 2)

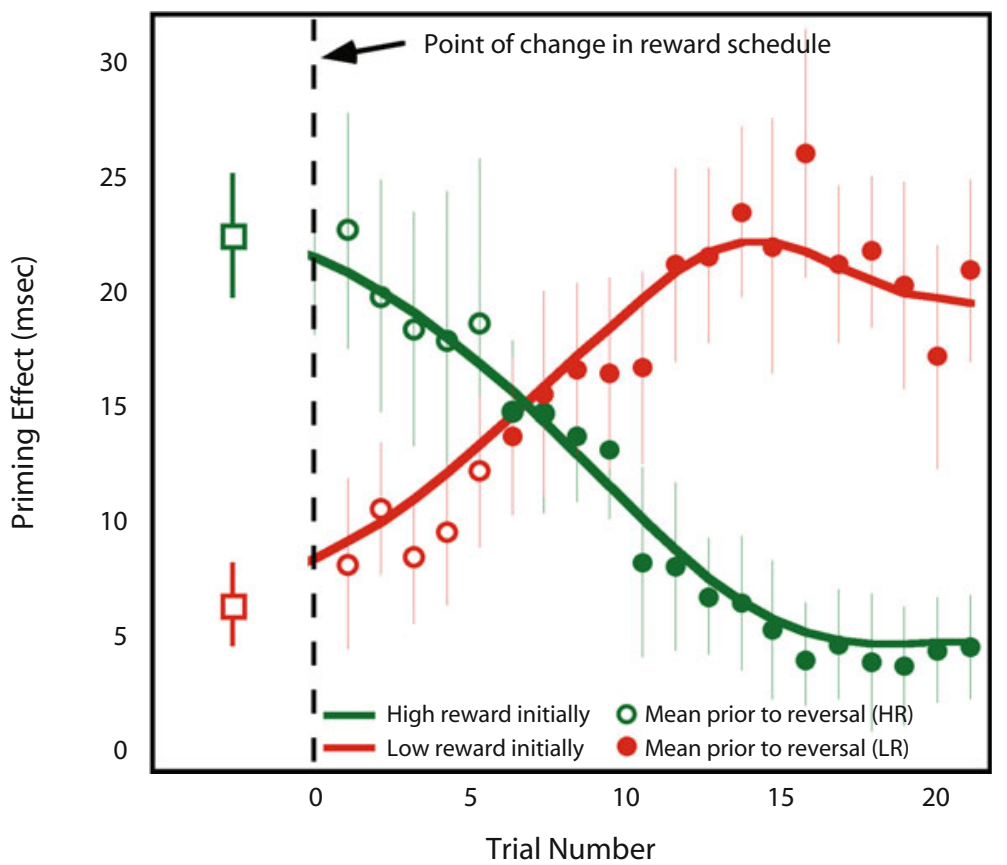

Figure 4. (A) How inverse efficiency is affected by unpredictable changes in reward schedule across successive trials. At the far left, the two square symbols indicate average inverse efficiency across 8 trials (not shown separately) preceding a reversal in the reward schedule. This reversal occurs at trial "zero," as indicated along the $x$-axis and by the vertical dashed line. Mean inverse efficiency is then plotted for each of 20 successive trials following the reward schedule reversal. The target color that was likely (75\%) to yield a high reward prior to reversal is (arbitrarily) shown in green (dark gray in printed edition of this article), and the target color likely (75\%) to yield a low reward prior to the reversal is shown in red (light gray in print). (The actual target colors were equally likely to play either role in the experiment, with these being counterbalanced not only between participants, but also now within participants, due to the reversals in reward contingency.) The error bars show the $95 \%$ confidence intervals around each point. The symbols become filled when there is no longer any overlap between pre- and postreversal confidence intervals, meaning that performance has significantly $(\alpha=.05)$ changed from the prereversal level, as starts to become evident from the sixth trial after a reward reversal. (B) Analogous outcome for trial-to-trial target repetition effects, with the $y$-axis now denoting differences in inverse efficiency between nonrepeat minus repeat trials. Again, a reversal in reward contingencies has a significant impact on this measure from the sixth trial after such a reversal. The solid lines show a nearest neighbor fit where the value $\left(x^{\prime}\right)$ of the fitted line at each point $(n)$ on the abscissa is determined by $x_{n}^{\prime}=\left[\left(x_{n-2}\right)+\left(x_{n-1}\right)+x_{n}+\left(x_{n+1}\right)+\left(x_{n+2}\right)\right] / 5$, shown here as the stabilized estimates following three iterations of this fitting algorithm. Thus we averaged over a 5-trial window, centered on $\left(x_{n}\right)$, iterating this smoothing procedure three times to obtain the fitted line. Each point shown is the interparticipant mean of individual means, prior to any smoothing. 
search behavior. One possible neural source for the present effects might be that that the higher rewarded color leads to stronger (or more enduring) corresponding neural activity, facilitating processing of that same target feature on a subsequent trial (see Kristjánsson, 2008; Kristjánsson \& Campana, 2010; Kristjánsson et al., 2007). This possibility might now be examined by applying measures of neural activity to the new procedures we have introduced here.

Our results from human pop-out search have interesting parallels with recent work on the effects of reward in saccadic choice by nonhuman primates (for reviews, see, e.g., Sugrue, Corrado, \& Newsome, 2005; Trommershäuser, Glimcher, \& Gegenfurtner, 2009). Sugrue et al. (2004) found that monkeys adjusted saccadic choices rapidly to unpredictable changes in reward schedules, reporting trial-by-trial effects that extended further than just the immediately preceding trial (see also Lau \& Glimcher, 2005). They also observed that neurons in the lateral intraparietal area showed activity modulations that closely mirrored the behavioral pattern (see also Dorris \& Glimcher, 2004; Platt \& Glimcher, 1999). A recent human fMRI study (Kristjánsson et al., 2007) showed that target repetition effects in a search paradigm similar to that used here (but without the present reward manipulations) also cause activity modulations in the intraparietal cortex. This suggests intriguing avenues for future research into the relationship between attentional deployment, motivational factors, and the underlying neural circuitry.

\section{AUTHOR NOTE}

Portions of this research were presented at the September 2009 meeting of the European Society for Cognitive Psychology in Krakow, Poland. A.K. was supported by grants from the University of Iceland Research Fund and the Human Frontiers Science Program; J.D. by the Wellcome Trust and a Royal Society Anniversary Research Professorship. We are grateful to Gianluca Campana, Riccardo Pedersini, Jeremy Wolfe, and an anonymous referee for insightful comments and suggestions for improvement during the review process. Correspondence concerning this article should be addressed to Á. Kristjánsson, School of Health Sciences, University of Iceland, Aragata 14, 101 Reykjavik, Iceland (e-mail: ak@hi.is).

\section{REFERENCES}

BeCKER, S. I. (2008). The stage of priming: Are intertrial repetition effects attentional or decisional? Vision Research, 48, 664-684.

Bravo, M. J., \& NAKAYAMA, K. (1992). The role of attention in different visual-search tasks. Perception \& Psychophysics, 51, 465-472.

Campana, G., Cowey, A., \& Walsh, V. (2002). Priming of motion direction and area V5/MT: A test of perceptual memory. Cerebral Cortex, 12, 663-669.

Campana, G., Cowey, A., \& Walsh, V. (2006). Visual area V5/MT remembers "what" but not "where." Cerebral Cortex, 16, 1766-1770.

Della Libera, C., \& Chelazzi, L. (2006). Visual selective attention and the effects of monetary rewards. Psychological Science, 17, 222227.

Dorris, M. C., \& Glimcher, P. W. (2004). Activity in posterior parietal cortex is correlated with the relative subjective desirability of action. Neuron, 44, 365-378.

Fecteau, J. H. (2007). Priming of pop-out depends upon the current goals of observers. Journal of Vision, 7(6, Art. 1), 1-11.

Geng, J. J., Eger, E., Ruff, C. C., Kristuánsson, Á., Rotshtein, P., \& Driver, J. (2006). On-line attentional selection from competing stimuli in opposite visual fields: Effects on human visual cortex and control processes. Journal of Neurophysiology, 96, 2601-2612.

Geyer, T., Müller, H. J., \& Krummenacher, J. (2006). Cross-trial priming in visual search for singleton conjunction targets: Role of repeated target and distractor features. Perception \& Psychophysics, $\mathbf{6 8}, 736-749$

Goolsby, B. A., \& SUZUKI, S. (2001). Understanding priming of colorsingleton search: Roles of attention at encoding and "retrieval." Perception \& Psychophysics, 63, 929-944.

Hickey, C., \& Theeuwes, J. (2008). Reward primes visual search [Abstract]. Perception, 37(Suppl.), 46a.

Ivry, R. B., \& Cohen, A. (1990). Discrimination of short- and longrange apparent motion in visual search. Journal of Experimental Psychology: Human Perception \& Performance, 16, 317-331.

JuLEsz, B. (1984). A brief outline of the texton theory of human vision. Trends in Neurosciences, 7, 41-45.

Kiss, M., Driver, J., \& Eimer, M. (2009). Reward priority of visual target singletons modulates event-related potential signatures of attentional selection. Psychological Science, 20, 245-251.

Kristuánsson, Á. (2006). Rapid learning in attention shifts: A review. Visual Cognition, 13, 324-362.

KRISTJÁnsSON, Á. (2008). "I know what you did on the last trial"A selective review of research on priming in visual search. Frontiers in Bioscience, 13, 1171-1181.

KRISTJÁNSSON, Á. (2009). Learning in shifts of transient attention improves recognition of parts of ambiguous figure-ground displays. Journal of Vision, 9(4, Art. 21), 1-11.

Kristjánsson, Á., \& Campana, G. (2010). Where perception meets memory: A review of repetition priming in visual search tasks. Attention, Perception, \& Psychophysics, 72, 5-18.

KristuÁnsson, Á., \& Driver, J. (2008). Priming in visual search: Separating the effects of target repetition, distractor repetition and rolereversal. Vision Research, 48, 1217-1232.

Kristjánsson, Á., IngVarsdótTir, Á., \& Teitsdóttir, U. D. (2008). Object- and feature-based priming in visual search. Psychonomic Bulletin \& Review, 15, 378-384.

Kristjánsson, Á., Mackeben, M., \& Nakayama, K. (2001). Rapid, object-based learning in the deployment of transient attention. Perception, 30, 1375-1387.

Kristuánsson, Á., \& Nakayama, K. (2003). A primitive memory system for the deployment of transient attention. Perception \& Psychophysics, 65, 711-724.

Kristuánsson, Á., SigurJónsdótTir, Ó., \& Driver, J. (2009, September). The effects of financial reward schedules on repetition priming in visual search. Poster presented at the 16th Meeting of the European Society for Cognitive Psychology, Krakow, Poland.

Kristuánsson, Á., Vuilleumier, P., Malhotra, P., Husain, M., \& DrIVER, J. (2005). Priming of color and position during visual search in unilateral spatial neglect. Journal of Cognitive Neuroscience, 17, 859-873.

Kristuánsson, Á., Vuilleumier, P., Schwartz, S., Macaluso, E., \& DrIVER, J. (2007). Neural basis for priming of pop-out revealed with fMRI. Cerebral Cortex, 17, 1612-1624.

Lamy, D., Antebi, C., Aviani, N., \& Carmel, T. (2008). Priming of pop-out provides reliable measures of target activation and distractor inhibition in selective attention. Vision Research, 48, 30-41.

Lau, B., \& Glimcher, P. W. (2005). Dynamic response-by-response models of matching behavior in rhesus monkeys. Journal of the Experimental Analysis of Behavior, 84, 555-579.

Maljkovic, V., \& Nakayama, K. (1994). Priming of pop-out: I. Role of features. Memory \& Cognition, 22, 657-672.

Maljkovic, V., \& Nakayama, K. (2000). Priming of popout: III. A short-term implicit memory system beneficial for rapid target selection. Visual Cognition, 7, 571-595.

Müller, H. J., Heller, D., \& Ziegler, J. (1995). Visual search for singleton targets within and across feature dimensions. Perception \& Psychophysics, 57, 1-17.

MÜLLER, H. J., \& KRUmmenacher, J. (2006). Visual search and selective attention. Visual Cognition, 14, 389-410.

Neisser, U. (1967). Cognitive psychology. New York: AppletonCentury-Crofts.

Olivers, C. N. L., \& Meeter, M. (2006). On the dissociation between compound and present/absent tasks in visual search: Intertrial priming is ambiguity driven. Visual Cognition, 13, 1-28.

Platt, M. L., \& Glimcher, P. W. (1999). Neural correlates of decision variables in parietal cortex. Nature, 400, 233-238. 
Shen, J. Y., \& ChUn, M. M. (2009). Reward modulation of search and priming of pop-out [Abstract]. Journal of Vision, 9(8), 1213.

Sigurdardottir, H. M., Kristuánsson, Á., \& Driver, J. (2008). Repetition streaks increase perceptual sensitivity in brief visual search displays. Visual Cognition, 16, 643-658.

Sugrue, L. P., Corrado, G. S., \& Newsome, W. T. (2004). Matching behavior and the representation of value in the parietal cortex. Science, 304, 1782-1787.

Sugrue, L. P., Corrado, G. S., \& Newsome, W. T. (2005). Choosing the greater of two goods: Neural currencies for valuation and decision making. Nature Reviews Neuroscience, 6, 363-375.

Townsend, J. T., \& Ashby, F. G. (1983). Stochastic modeling of elementary psychological processes. New York: Cambridge University Press.

Treisman, A. M., \& Gelade, G. (1980). A feature-integration theory of attention. Cognitive Psychology, 12, 97-136.

Trommershäuser, J., Glimcher, P. W., \& Gegenfurtner, K. R. (2009). Visual processing, learning and feedback in the primate eye movement system. Trends in Neurosciences, 32, 583-590.

Van der Stigchel, S., Belopolsky, A. V., Peters, J. C., Wijnen, J. G.,
Meeter, M., \& Theeuwes, J. (2009). The limits of top-down control of visual attention. Acta Psychologica, 132, 201-212.

Wang, D., Kristuánsson, Á., \& NaKayama, K. (2005). Efficient visual search without top-down or bottom-up guidance. Perception \& Psychophysics, 67, 239-253.

Wang, Q., Cavanagh, P., \& Green, M. (1994). Familiarity and pop-out in visual search. Perception \& Psychophysics, 56, 495-500.

Wolfe, J. M. (1998). Visual search. In H. Pashler (Ed.), Attention (pp. 13-73). Hove, U.K.: Psychology Press.

Wolfe, J. M., Cave, K. R., \& Franzel, S. L. (1989). Guided search: An alternative to the feature integration model for visual search. Journal of Experimental Psychology: Human Perception \& Performance, 15, 419-433.

Wolfe, J. M., \& Horowitz, T. S. (2004). What attributes guide the deployment of visual attention and how do they do it? Nature Reviews Neuroscience, 5, 1-7.

(Manuscript received November 17, 2009; revision accepted for publication February 7, 2010.) 